\title{
Activity model and computer aided system for defining sheet metal process planning
}

\author{
J. Ciurana ${ }^{\mathrm{a}, *}$, I. Ferrer $^{\mathrm{b}}$, J.X. Gao ${ }^{\mathrm{c}}$ \\ ${ }^{a}$ Department of Mechanical Engineering and Industrial Construction, University of Girona, Spain \\ ${ }^{\mathrm{b}}$ Department of Business Administration, Management and Product Design, University of Girona, Spain \\ ${ }^{\mathrm{c}}$ Department of Enterprise Integration, Cranfield University, United Kingdom
}

\begin{abstract}
This paper focuses on the problem of choosing the manufacturing route and characteristics in sheet metal processes, a very important element in computer aided process planning (CAPP) systems. At present, decisions concerning the operations, die, machine and process parameters used in sheet metal are based on experience.

One of the objectives of this work has been to develop an activity model to help define sheet metal processes. This activity model allows focusing on the second objective, which is to implement a computer aided system to select and define the parameters of the process definition in the case of drawing operations with sheet metal. The result is the selection of parameters related to the operations chosen, the kinds of operations, the sequence of these operations and the lay-out die dimensions for the product.

A range of parts were evaluated. They were chosen because they were considered to be representative cases. The results obtained by the system are compared with the values proposed in reference manuals, and by experienced experts. The work has served to determine how to adjust the computer aided system.

Applying the method helps to make the right decisions about the sheet metal operations related to drawing processes. The experiments have led to a reduction in processing times.
\end{abstract}

Keywords: Sheet metal processes; Computer aided process planning; Activity model; Drawing processes; Decision support system

\section{Introduction: process planning}

Process planning is the act of preparing detailed operating instructions for turning an engineering design into an end product, i.e. the part. This implies the need to translate the design specifications of a part into the required manufacturing operating instructions, to convert it from the raw material to the part in its final state [1].

There is a great deal of manufacturing data involved in process planning, such as the identification of machines, tools, flanging, parameters selection for the process, operations, etc. [2]. All of this data has to be evaluated in order to select the sequence of operations that will make up what is known as the

* Corresponding author at: Department of Mechanical Engineering and Industrial Construction, University of Girona, Av. Lluís Santaló s/n, 17071 Girona, Spain. Tel.: +34 972 419822; fax: +34 972418098.

E-mail address: quim.ciurana@udg.es (J. Ciurana). sheet route. The sequence is generally made to conform with particular objectives, such as, for example, the shortest time and/or the minimum cost.

Process planning requires many kinds of human abilities, which should be present in the process planner [1].

The traditional approach to resolving the process planning task is the one commonly used in a manufacturing company; the plans are handed over to the manufacturing process experts who then specify the procedures to make the product. The process planners, using their experience and knowledge, generate instructions for manufacturing the products based on the design specifications and the available installations and operators. The fact that there are few experienced process planners and that, when faced with the same problem, different process planners would probably come up with different plans is an indication of the heterogeneity that exists in process planning [1]. Consistent and correct planning requires two things: knowledge of manufacturing processes and experience [3]. This has led to the 
development of computer aided process planning (CAPP) systems, which are becoming more and more important in this field.

The most maturely developed area so far has been focused on machining applications. Research and development in manufacture applications such as heat treatment, forging, injection moulding, and sheet metal manufacture is still premature, and the reported systems for sheet metal manufacture rely on a high level of interaction from the expert who provides the decisionmaking at different stages of planning [4].

Sheet metal components are widely used in various industries like aerospace, electronics, machine tools, refrigeration and air conditioning, etc., and they form a significant part of manufacturing activity. Sheet metal components are important not only from a functional point of view, but also from an aesthetic point of view, since they are used as enclosures to cover products and are visible to the outside world. These components vary in size, shape and complexity. Therefore, sheet metal processes receive a lot of attention and are widely used by the metal working industries [3].

The manufacturing processes required for sheet metal components are identified by analyzing the component layout, and then design information is manually translated into manufacturing information [5]. To overcome inherent difficulties and limitations associated with human beings, research work is progressing into the area of automatic transformation of design information into manufacturing information through feature recognition [3].

The main aim of this research is to develop a computer aided system for sheet metal manufacture. The purpose of the system developed is to calculate the manufacturing parameters needed for drawing components to be produced. The idea is not to replace the role of expert process planners, but instead, to provide a tool to assist them to be more efficient and consistent in their work.

This project was set up in collaboration with a manufacturing company. In the company sheet metal forming processes such as bending and drawing are used to manufacture some components. Further development of these processes will allow the user to enter the product data as a feature for simple sheet metal components. Future developments in the system will make it capable of coping with the remaining, more complex components and possibly of being modified for prismatic and other rotational parts.

The initial stage of the research was conducted on-site at the company, in order to understand the manufacturing processes and process planning tasks, and to find out the user requirements for computer aided process planning functions. The second stage was to develop the first iteration of the computer aided system. The underlying methodology can also be tailored to general sheet metal fabrication.

The goal of this work is to contribute to developing a tool to help the planning process applied to sheet metal processes. This work focuses on drawing processes. The work helps the engineer to decide on manufacturing parameters such as the operations sequence and the die dimensions for a drawing sheet metal process. This work is based on the knowledge obtained from the collaboration with the enterprises.
The future proposal will be a tool which is capable of defining a route sheet without depending on the sheet metal processes it is considering. The work will cover different processes such as flanging, blending, punching, trimming, drawing, etc. The integration of future recognition starting on CAD systems is necessary. There has been no work which covers this so far. However, to obtain this goal the research should start with a more concrete objective, which is presented in this paper.

\section{CAPP systems}

CAPP systems are beginning to be developed as a link between design and manufacturing, filling the existing gap between CAD (computer aided design) and CAM (computer aided manufacturing), along with the need for MRP (material requirement planning) to work with standard and optimised routes which can be used in schedule production [2]. Based on the CIM strategy, CAPP allows the user to develop an integrated structure that deals with the flow of information between CAD, CAPP, MRP and numeric control (NC) activities within the company [6], as shown in Fig. 1.

CAPP systems have evolved from the traditional/manual approach into two recognised approaches: the variant approach and the generative one.

The traditional or manual approach means examining the drawing of the engineering part and developing plans and instructions for the manufacturing processes based on an understanding of the machines, the tools, the materials, the related costs and the working practices of the company.

The variant approach is based on getting the plan of a similar process carried out previously and modifying it. In these kinds of CAPP systems, the parts are sorted into groups for which process plans are made that are then stored in the computer to be recovered as required for new parts or for modifications. In this case, Group Technology helps to identify a suitable group to which the new part can belong. The main disadvantage of this is that the quality of the process planning still depends on the prior knowledge of the process planner [1,6].

The systems using the generative approach are designed to automatically draw up an individual plan for each part, using the appropriate algorithms that process the information in the manner required by the decisions that need to be taken. The first versions of these generative systems used tables and decision trees to represent the logic of manufacturing [7].

The result, i.e. the output of a CAPP system, is a route sheet. The route sheet is the sequence of manufacturing operations which contains the details of the process. This sequence has to be the optimal sequence, and is obtained from the defined objectives, but even so, the optimum process plan may not guarantee the best way of manufacturing the part in the plant at a specific moment, as it could lead to the overloading or under use of some machines with subsequent bottle-necking. For this reason, the generation of a single plan is not recommended [8].

Different researchers have proposed different sequential schemes with regard to the steps taken by a computer aided planning system. All of these systems have been programmed and implemented, but performance has been poor, especially because 


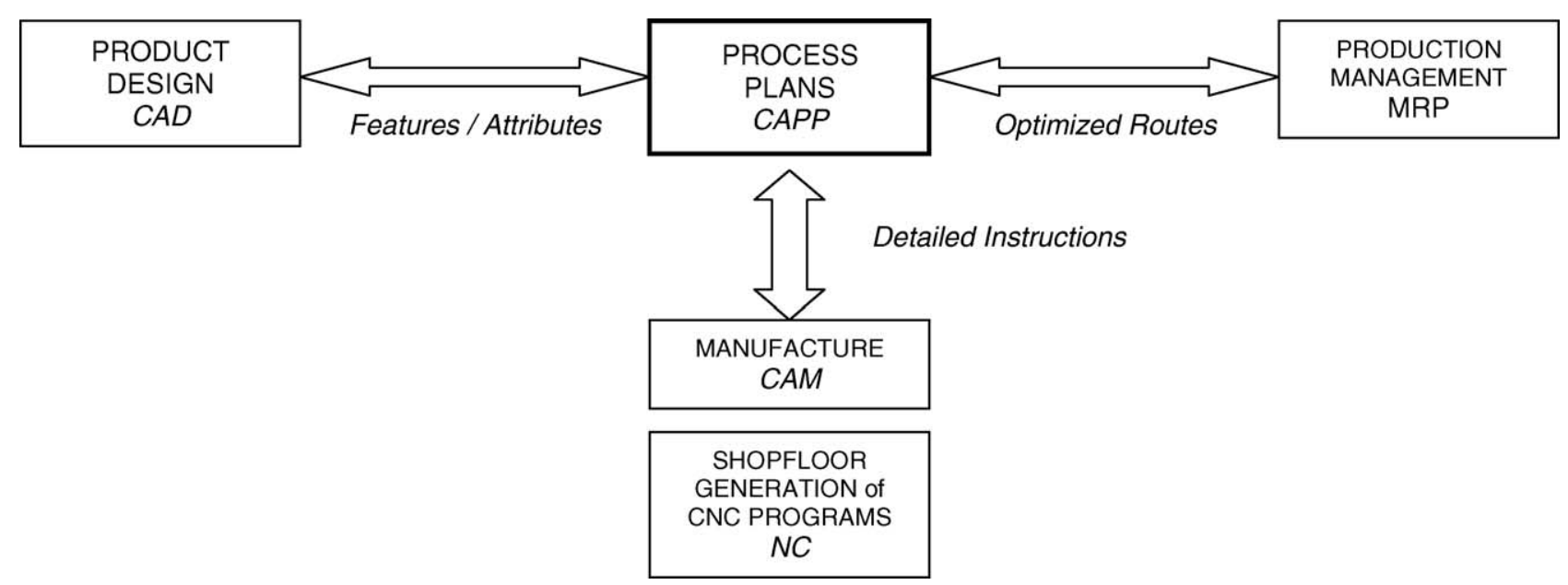

Fig. 1. The integration of CAPP systems in the production environment.

they require lengthy calculation times, not only due to the multiple routes planned for each piece, but also for the quantity of operations needed to optimise the manufacturing parameters for each one [8].

\section{Research design and methodology}

It is advisable to make initial constraints that it will be explained before describing the methodology used; there are four constraints to consider:

1. Firstly it has to consider the selection of the parts to include in the study. Obviously, it could not carry out field work on all the possible parts that might be developed in the sector of the study. The rotational part is chosen because rotational parts come from the enterprises which collaborated in the knowledge stage. Different works already developed by the authors started with this kind of feature and the results obtained were good [8]. For this reason, it is believed that it can extrapolate useful information from the future results for the majority of the parts currently being manufactured by the industry.

2. Secondly, it has to consider the number of sheet metal processes and operations to study [9]. For the same reasons the processes in the study are limited, the number of operations are limited to those commonly used in the companies studied and related with drawing or deep drawing operation.

3. Thirdly, to understand the sheet metal processes an activity model is developed. The activity model allows detecting the flow analysis for these kinds of processes. The flow analysis of the process represents the information between different phases in the activity model. In particular, it considers the drawing process. From the activity model it is obtained the phases in which manufacturing processes are divided: blank decision, process definition, die/tool definition, machine selection and manufacture.

4. Fourthly, in order to start the implementation it has to consider one phase of the whole manufacturing process. The first phase is blank decision; however, work on this phase has been developed by the same authors in other works not yet published. Therefore this work will use the next phase, i.e. process definition.

5. Finally, it is considered that, within the framework of assisted process planning, it is essential to give the computer applications a user-friendly interface that helps to reduce errors when the user introduces data. In addition, the application needs should be capable of detecting typographical errors and interacting with the user, providing information on the current calculation stage of the algorithm, detecting anomalies or incoherent situations, and giving help about what to do if such situations arise.

The steps used in the methodology are explained in the light of the stated objective and the considerations outlined in the previous section. The proposed scheme is divided into three parts: knowledge acquisition, the activity model and development and implementation of the tool. It is also necessary to outline the tasks that have enabled to develop it in order to show clearly the principles behind it and its scope.

\subsection{Knowledge acquisition}

After a thorough analysis of sheet metals processes, beginning with Radhakrishnan [9] and Choi [10], the research obtained a complete list of all the operations that are related with drawing processes. This analysis takes into account the influence of the geometrical features of the part on the calculation of the manufacturing parameters, as well as the correlations between these factors.

The technical factors, consisting in the different constraints of technology, the geometry, the material and the machine, have input roles in the process and the future algorithm, and provide results in the drawing operation. These values are needed in order to select the route sheet.

Other factors are those derived from the close relationship existing between the calculations during the decision process and the factors mentioned above.

The system has been developed, improved and expanded in a number of stages. At each stage feedback from process planners 
has been collected and carefully considered for the next stage. One of the most important tasks is to gather manufacturing knowledge which is used to develop the computer system. The computer system contains all the drawing process characteristics and the logic that determines which parameter processes and die dimensions to use in order to choose the correct machine. This is effectively based on the company's 'know-how'.

The first stage was to carry out different elicitations with the enterprises interested in developing the tool. These enterprises collaborated in defining and adapting theoretical scientific manufacturing factors into practical ones, and also establishing which parameters should be the main ones. The work started by understanding the 'know-how' of these enterprises interested in the aided tool that is being developed in the present work.

To do this the research began by arranging brainstorming meetings with the company's process planners and other relevant people. All the factors affecting the decision-making and all the drawing processes used for producing the components were collected. At the initial stage of the brainstorming sessions normal brainstorming rules were applied, in which no analysis, filtering, review or classification of the ideas was allowed, thereby keeping up the pace and not inhibiting unusual thoughts.

All the information collected on the scientific manufacturing process $[9,11]$ works was checked by comparing it with the information used by the enterprises. Some of the information is used in the same way, however, there was some information that has never been used or applied in the different ways that were suggested. The elicitation was used in order to correct some enterprises' defects and improve their processes.

The second stage in understanding the knowledge of the enterprises started with the factors previously identified being reviewed and arranged into logical flowcharts. The developed logic represents the computer system and this document was distributed to all participants. Any disagreements on the points were raised in open discussions and solutions to the disagreement were found. The computer system and relevant manufacturing information was entered into the initial version of the prototype system and tested on some component parameters, which were then compared with the generated process plans.

This comparison between the literature and the technology in use makes the information used in the present and future works more accurate and appropriate.

The information has been obtained and managed in order to define an activity model usually used by the engineers and the workers. An IDEF0 model was developed as the paper will explain later, and was generated from a generic activity (Figs. 2 and 3).

\subsection{Activity model}

Having identified the factors to be considered in order to obtain a sequence decision capable of meeting the described objectives, the research went on to analyse the manufacturing activity in Fig. 3 and the drawing operations in more detail. From this study the following steps stand out:

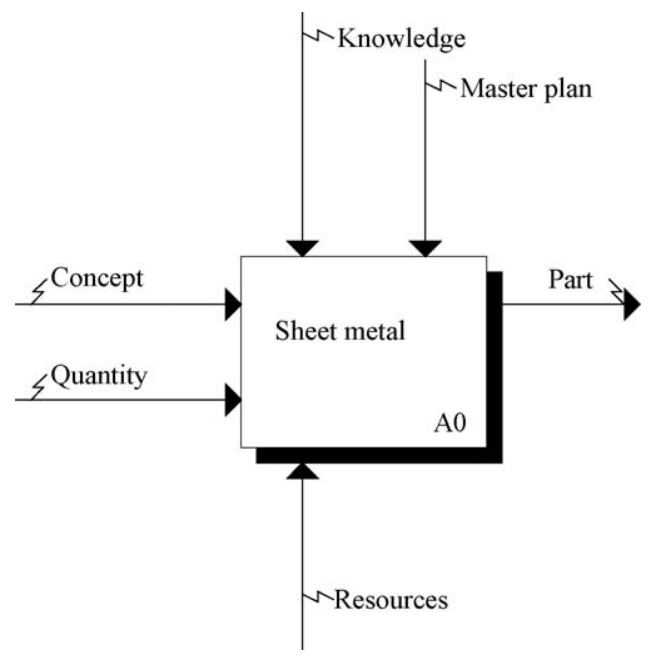

Fig. 2. Principal node of the activity model.

a. Beginning with an exhaustive study on the types of geometrical shapes existing in sheet metal features [3], the dimensional features necessary in order to study all the cases that might arise are defined. As mentioned in the initial constraints, the evaluation of the features defined was carried out on a representative sample provided by the enterprise from which the research obtained information.

b. Directly from this analysis, the complete activity model (Fig. 4) that allowed introducing this aspect into the algorithm being designed is obtained. The IDEF0 model allows detecting the existing interrelations between activities.

- A21 (blank decision) this step determines the dimensions and the form of the blank (material at the first stage) based on the dimensions and the geometrical form of the part. This is the first stage so the information comes from the CAD system.

- A22 (process definition) this is based on the drawings and quantities needed to manufacture the part. The process is defined and the definition consists in determining the die required for the sheet metal process. The dimensions of the

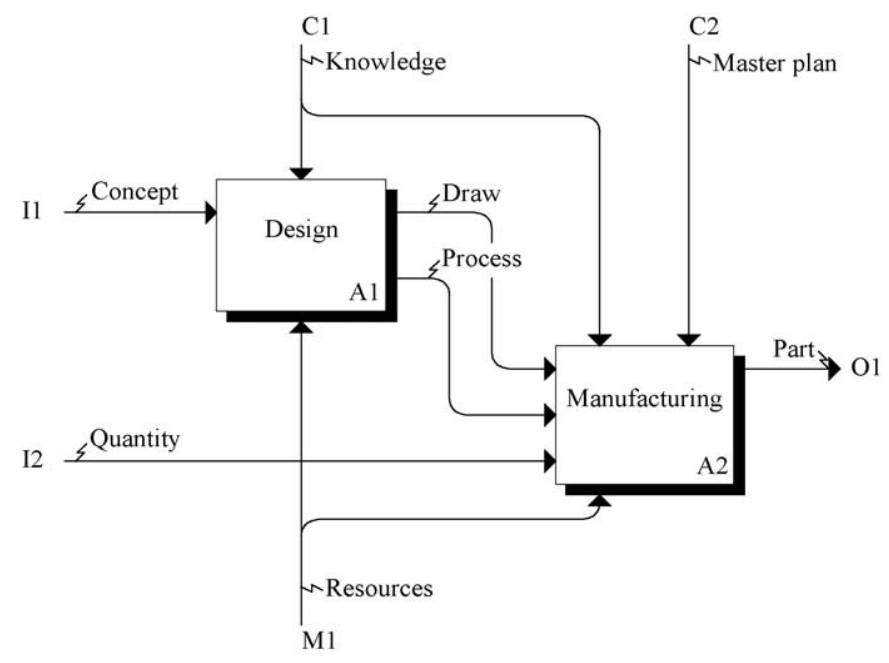

Fig. 3. First layer of the activity model. 


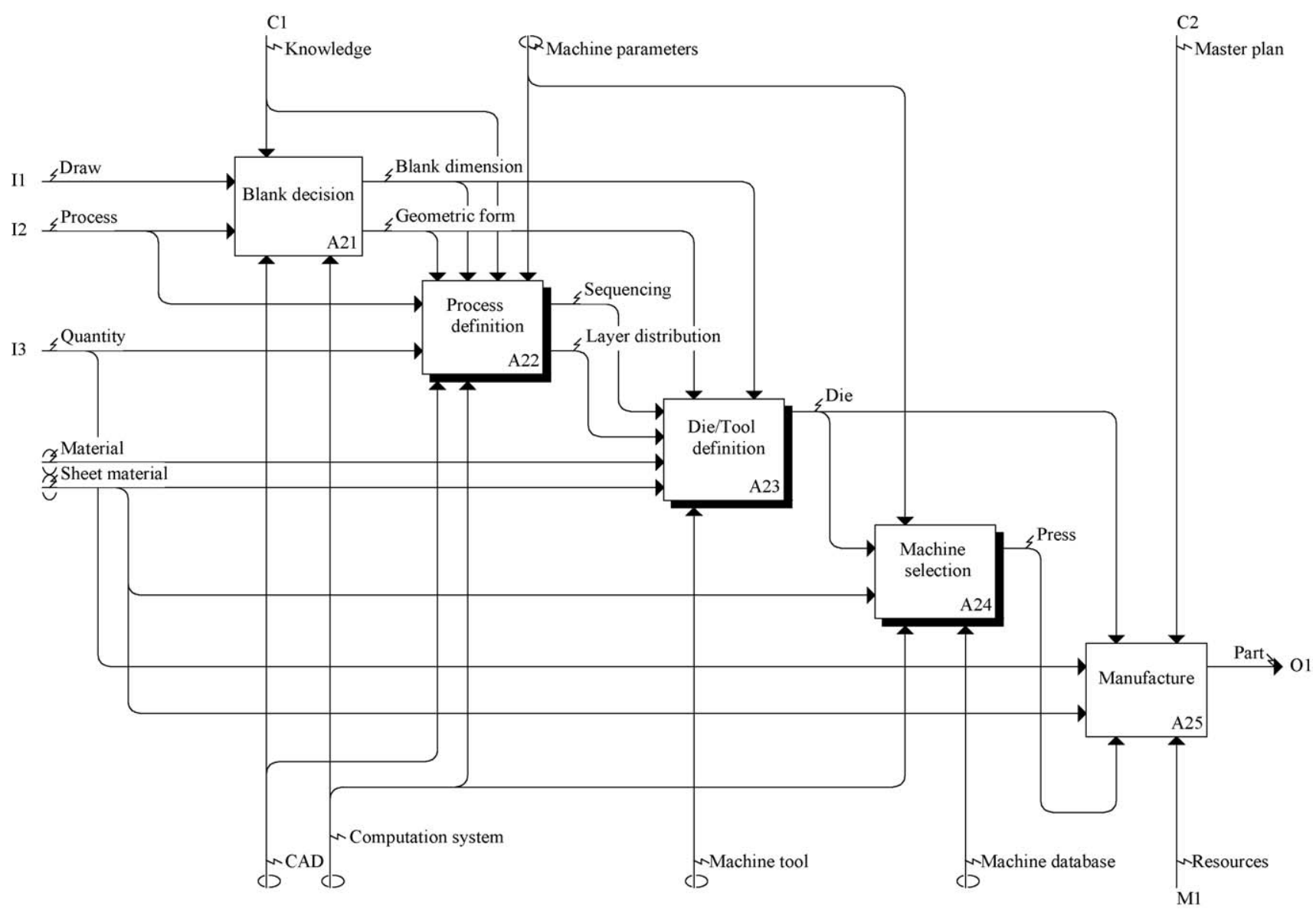

Fig. 4. Activity model A2.

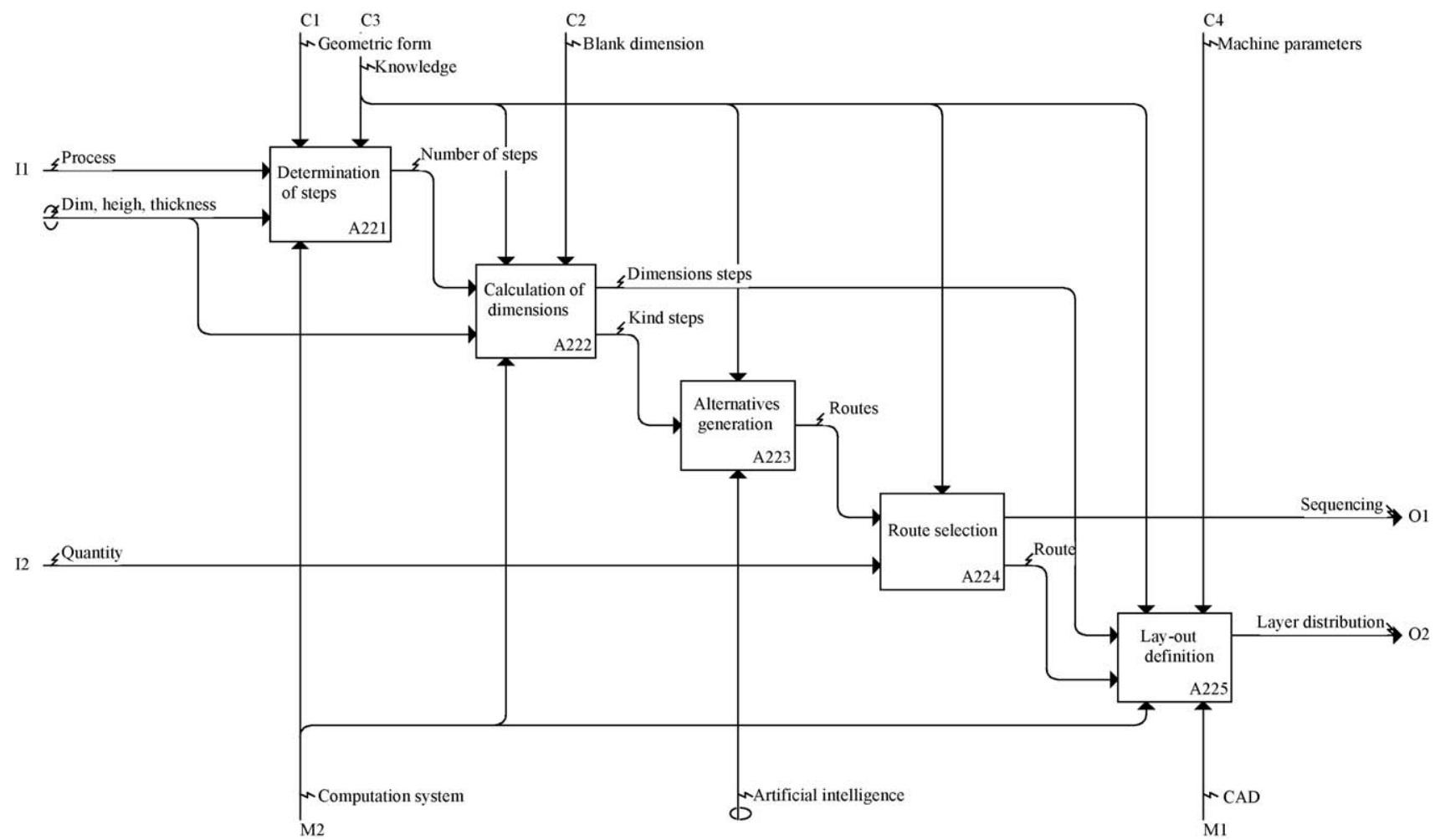

Fig. 5. Computerised part of the activity model. 
die are obtained. This decision allows deciding in the next steps on the machine to be used. This step also includes the sequence of the operations to obtain the part.

- A23 (die/tool definition) in this step the process to manufacture the die and tools are identified. Once the dimensions of the die are known it needs to be manufactured. This activity tries to define and identify the process of die manufacture.

- A24 (machine selection) in this stage the decision of which is the optimal machine to manufacture the part is made. This decision is based on the result of the A22 activity and the press working force required for the process. The machine selected should be capable and its capacity appropriate.

- A25 (manufacture) defines the real manufacture of the part. Once the machine and die are defined, the process is carried out by the free machine in the workshop.

The research work analysed the aspects of the process definition step in Fig. 4 that constrain the planning drawing process. The results obtained from this activity are shown more accurately in Fig. 5, which shows the activities carried out by the engineers and workers for the process definition in a real application. The process definition step is used to start the development of the computer application.

- A221 (determination of steps) this step determines the number of steps needed for the drawing process. The calculation is done based on different strategies. The input is the drawing of the part, its dimensions and form.

- A222 (calculation of dimension) this provides the different dimensions of width and height for each step of the drawing process. This activity can also define and provide the operations needed to manufacture the part.

- A223 (alternatives generation) this provides different route sheet alternatives. These alternatives are generated using efficiency and efficacy criteria. The route sheets can be modified. This activity allows creating the next route sheet process.

- A224 (route selection) provides the route sheet from the route sheet alternatives list. The route sheet selected is assigned to the process.

- A225 (lay-out definition) die dimensions are defined using data collected in the A221 activity and the different calculations done in previous activities. The form and the sequence operations on the die are defined too. The activity also allows replacing some security parameters. With this modification the die dimensions change, and the activities show the result.

\subsection{Development and implementation of the tool}

The software tool used for the prototype system can be regarded as an application program, which consists in the following components: a high level language, a user interface, a data language and an operating system.

An object-oriented programming language called Visual Basic was used to create the main functional modules. In the work, Visual Basic for the graphical user interface software, an application development environment, which is used for designing and developing the ground up large-scale, basic based, platform independent and distributed applications, is chosen. The operating platform chosen was Windows 2000 running on a personal computer, but this platform can be changed for other more current versions of Windows.

The developed prototype system provides two options for the geometry of the process definition. A simple drawing part and a drawing part with a flanging bend that is not deformed. The algorithm and the program are the same; only the dimensions of the flanging controls are different, introducing the dimension or a 0 .

The computer system starts with the geometrical definition of the part. Based on these geometrical dimensions the program executes the calculations of the processes parameters in order to obtain the result. The result gives the parameters for the die and a proposal for the security dimensions which can be modified. If the security dimensions are modified the computer system can be executed once again in order to obtain the final solution. In the final stage of the process definition step, the operation numbers are generated, the route sheet selected and the die dimensions calculated, in increasing order and properly formatted for the screen and printer. The user can view the finished definition process parameters using the different Windows.

\section{Results and study findings}

Basically, the algorithm can be followed through five different phases. These coincide with the five activities generated in the activity model in Fig. 5 corresponding to the steps in the process definition activity.

\subsection{Establishing the steps for the drawing process}

The algorithm has to manage all the possible combinations of the initial variables (the constraints described in Fig. 6), the situations deriving from them can be changed during the computing process.

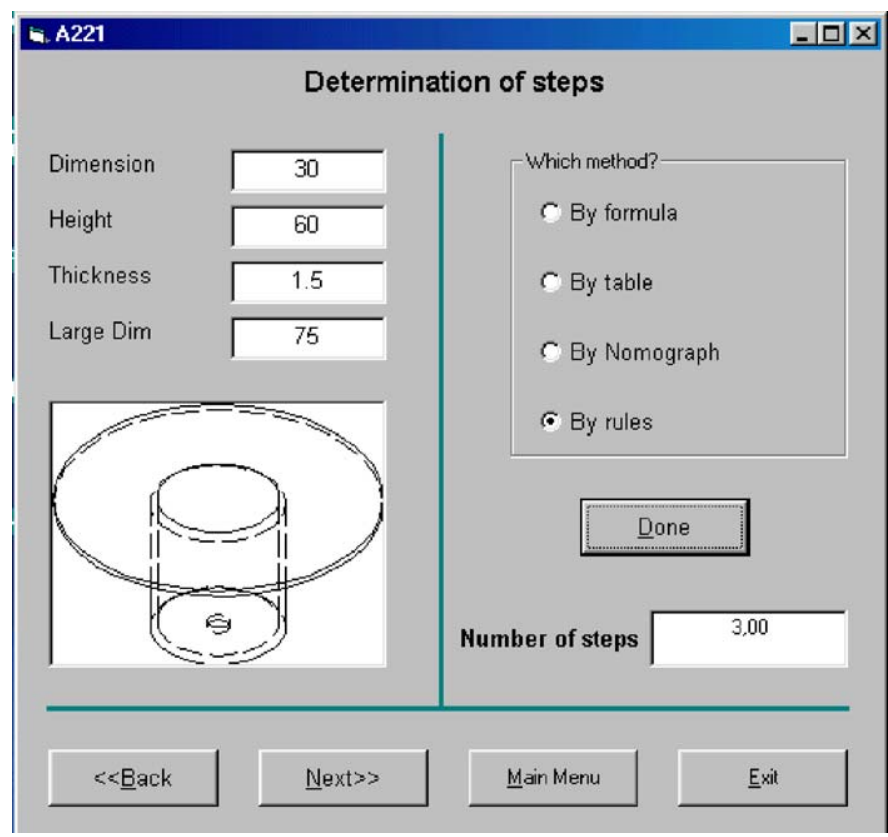

Fig. 6. Input, start procedure. 
In this stage an option button defines the drawing strategies [12] the algorithm implements to start the computation. Depending on the strategy the calculations will be different. The strategies are as follows:

- By formula, based on the application of the formula, which includes the diameter and the height.

- By table, based on the application of an information table, which represents averages of the relationship between the diameter and height.

- By nomograph, based on a graphic system like an abacus, which relates the diameter, the height and the width.

- By rules, based on experience rules, according to different cases in the enterprise.

Once the data is introduced, the algorithm calculates the number of steps needed for the drawing processes, as mentioned above this depends on the strategy chosen.

Then the prototype of the computer system is used. An engineering drawing of the part is the starting point for generating the process plan. The process planner identifies the geometrical and dimensional features from the drawings. $\mathrm{He}$ then enters the header information and the relevant component information into the system. The component information includes all the information required for generating the computation process definition such as diameter, height, width and clearances.

The system then starts the process definition phase to phase and the parameters are decided on as explained above. To do this, the system examines the information and extracts the appropriate operations depending on the stored manufacturing logic, the formula entered and activities introduced.

During this phase, the main goal is to determine the number of steps that the drawing process will take.

\subsection{Calculation of the dimensions of the steps}

This phase arises as a result of the last phase carried out. By means of a series of calculations, the algorithm determines the dimensions (height and diameter) for each drawing step determined in the above phase. The process of determining these dimensions requires each step to be of equal mass and the maximum reduction can be carried out in each step based on experimental studies.

Once the dimensions are calculated a drawing operation is carried out in the operations group for each step. Then, just as drawing operations are introduced into the process, the program also helps to add other operations needed for manufacturing the part, such as bending, trimming, and punching.

Finally, this Window calculates, on one hand, the different dimensions of diameter and height for each step of the drawing process and, on the other hand, it helps to decide the operations to carry out in order to manufacture the product, so the different operations needed to process the product are obtained.

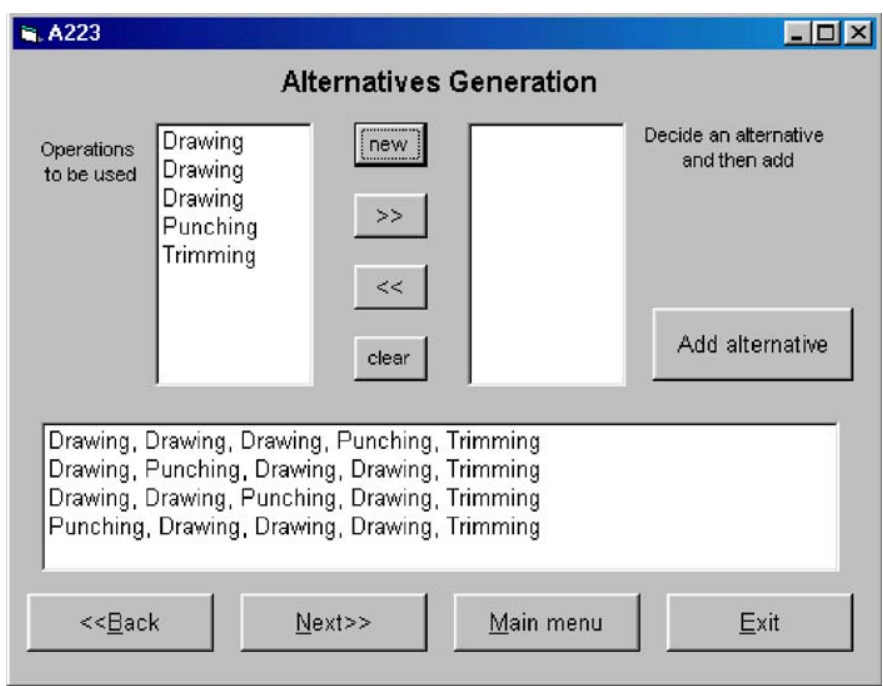

Fig. 7. The start and end list to generate route sheets.

\subsection{Generating alternatives}

Generating alternatives consists in creating a possible route sheet. Every route sheet is created using the total of the operations ordered in different positions in a route sheet alternative. Each alternative is generated with logical, efficiency and efficacy criteria. There is a start, which contains all the operations needed. The possible route sheet list is filled out with the operations ordered in the route sheet generation (Fig. 7) and based on parameters established according to efficiency and efficacy criteria.

Each route sheet that is generated should be added to the list of route sheet generations, which contains all the alternatives generated.

Although the case of generating alternatives using a few operations is a relatively simple decision, the difficulty in introducing the knowledge rules becomes much greater. This is due to the fact that sometimes the use of experience is faster than the optimal solution. With this in mind, an open solution structure which, in the future with the implementation of an added database could allow to introduce more complex manufacturing knowledge and manufacturing science is created.

Finally, this phase of the computer system tries to generate a group of possible alternatives to manufacture the part.

\subsection{Route selection}

Once the route sheet group has been generated, the algorithm can go on to the subsequent phases with a reference of this group at its disposal, which will help in the selection task. All that is needed now is a decision on which route sheet to select.

This phase consists in selecting an alternative. The route sheet is chosen based on economical criteria. The aim of this step is to select a route sheet alternative. This simple phase is important due to its effect on the lay-out die definition, and therefore it will be important for the next phase. 
Up to this point, what it has described constitutes a common stage for manufacturing the part, except for the number of the steps decided on in the first phase. The result of this last computation is to lead the computer system to correspond to the different situations. However, beginning with the fact that stamping can be carried out by means of a single machine, the system will simplify the procedure and the process parameters will be calculated directly according to the diameters and heights of the corresponding expressions.

The different computations depend on different input data introduced at this phase. These inputs are related to the machine, clearances and the priorities of the operations.

Depending on the results of this phase, the system will go on to search for the machine options in the workshop. This computation is done in the A4 activity in Fig. 4, which introduces other parameters in the machine selection. Before providing the results obtained, these are first checked against the existing solutions, with particular attention to aspects such as the forces attained during the process, otherwise a new computation can be done. The computation is checked and readjusted and the necessary calculations carried out.

Finally, the aim of this phase is to calculate the lay-out dimensions of the die. It can be explained as the important dimensions of the die. These dimensions are the length dimension, width dimension and the feed rate or advance of the sheet metal between two operations.

\section{Empirical study}

Once the computation system that had been designed to meet the defined objectives was implemented on the computer, the research went on to apply it systematically to the representative sample described in Section 3 (initial constraints), to find out how efficient the system was and to what extent it met the aforementioned objectives.

An example of this application would be the calculation of the process definition parameters for the stamping process of the part shown in Fig. 8. The information about this operation is introduced into the first Window of the program (Fig. 6).

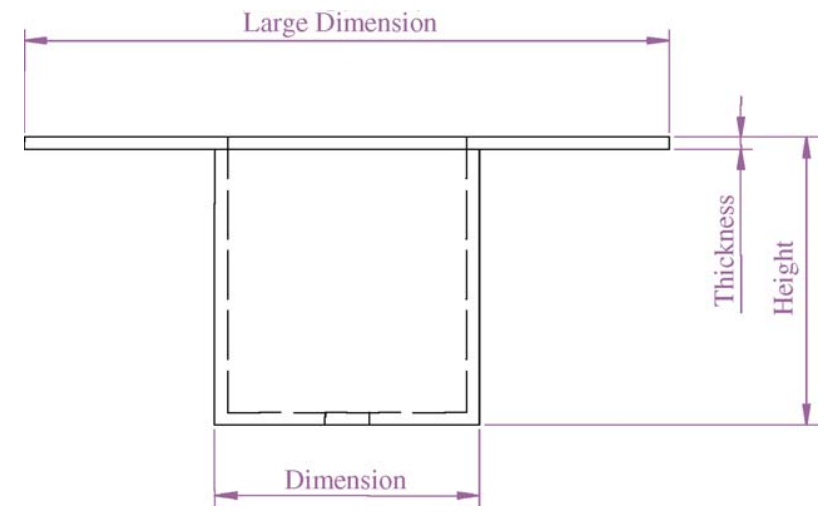

Fig. 8. Part studied.

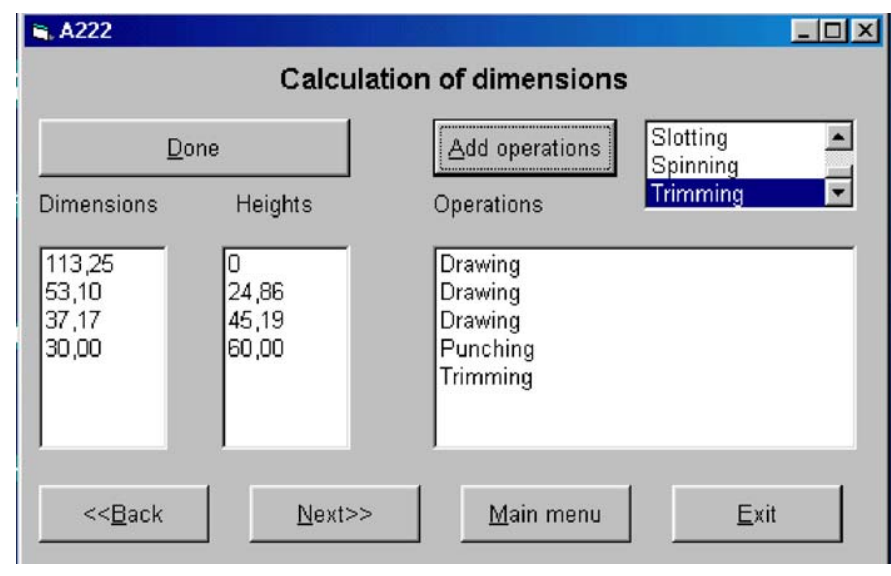

Fig. 9. Results of part X.

The proposal provided by an operations planner and following the usual methods would consist in carrying out the manufacturing process using four drawings at equal depths of $15 \mathrm{~mm}$, a punching process before the last drawing and another punching process at the end to cut the part out of the raw material. The die dimension needs space for at least six operations. This kind of route sheet was provided by the expert planners who the researchers worked with.

In contrast, three drawings are needed to fulfil the parameters suggested for the support system decision. The first one has a diameter of $53.10 \mathrm{~mm}$ and a $24.86 \mathrm{~mm}$ depth; the second a $37.17 \mathrm{~mm}$ diameter and a $45.19 \mathrm{~mm}$ depth; the third a $30 \mathrm{~mm}$ diameter and a $60 \mathrm{~mm}$ depth and finally, a punching process for the hole and trimming to cut the part from the raw material (Fig. 9). Only five operations are needed. This difference influences the die dimensions used.

A look at the machine dimensions and number of operations required to carry out the machining using these two alternatives, produces the following results:

- Operations planner proposal: $675 \mathrm{~mm} \times 120 \mathrm{~mm}$ and six operations.

- Computer system proposal: $535 \mathrm{~mm} \times 113 \mathrm{~mm}$ and five operations (Fig. 10).

Consequently, it could be said that in dimensional terms the result provided by conventional planning is $20.7 \%$ worse than that obtained through the algorithm. If the number of operations is considered this value is increased even more.

So, for this particular example, it could be said that using the computer system has a saving in the dimension of $20.7 \%$, not counting the subjective value of the number of operations which, bearing in mind that it has gone from six operations to five, would increase the percentage of time saved. In addition, the system takes into account the fact that the drawing conditions, demonstrated by Singh [11], have been met.

In each case using the computer system brings benefits in terms of reducing the number of operations, efficiency and efficacy of the route sheet and die dimensions of around $20 \%$. Nevertheless, in the different cases tested, the research found 


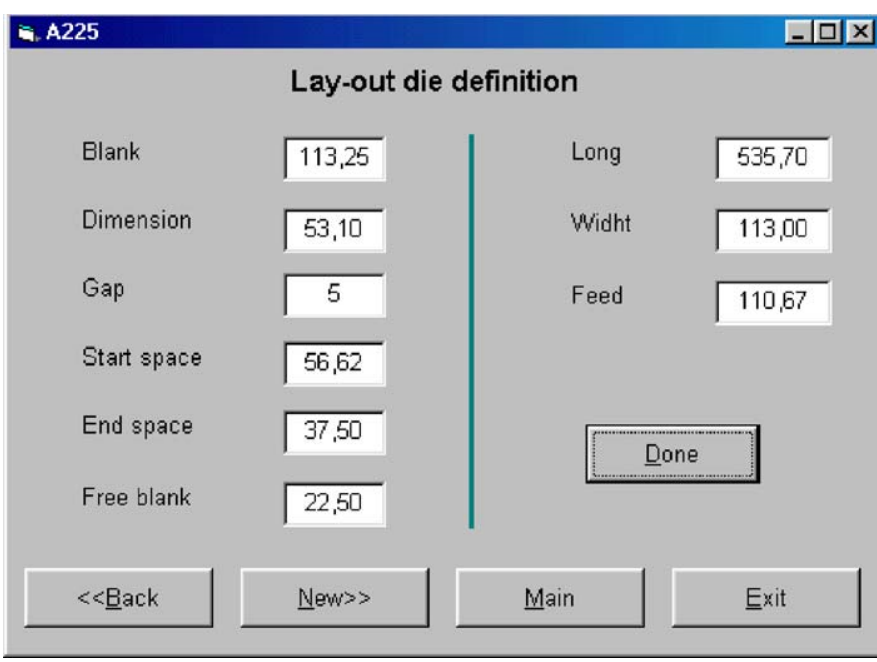

Fig. 10. Results of activity A224.

that, in addition to the dimensions saved in the die itself, the system also saves time in the planning process by up to $65 \%$ (assuming that in the non-assisted planning, the same level of optimisation provided by the algorithm is being sought). This is a considerable benefit, since the planner saves time and this has a positive effect in terms of repetitions and reducing wasted time and materials in the process.

\section{Discussions}

From the investigations it is found the difficult to take existing logical sequences for blending, punching or other sheet metal operations and apply them to drawing operations. The work also found that a need exists to design new optimisation strategies, better adjusted to the nature of these operations. The aim of this work is to contribute to filling this need.

One of the advantages of using this method is the time saved, up to $65 \%$, during the planning process. Having tested the system on real cases, this becomes a secondary advantage compared with the other advantages detailed below:

(a) Optimisation of the die dimensions, always respecting the technical specifications demanded by the product.

(b) The advantage of having a sequential flow that ensures that the system fulfils all the technical factors that have an influence on these kinds of operations; factors which are difficult to integrate into conventional planning techniques and are therefore often forgotten.

(c) The generation of a database that allows the system to easily obtain similar algorithms for other sheet metal operations, which is an important step forward in achieving the objectives of CAPP.

The presented application has been developed as a standalone system to avoid making any changes to the main planning system source code. The format for exchange of data is plain ASCII thereby permitting editing of the data using any word processor. The application was designed in a functional format with indi- vidual procedures to carry out separate tasks. These modules were individually tested for integrity to ensure that they carry out the specified task. This allows it to be used very easily in any Window's application.

The computer system also provides facilities for enlarging operations. It also has extensive search-and-replace operations. The procedure for loading the application into the planning system has been written in Basic. The data structure maintains a full list of the relationships between different characteristics of the product. The end file created provides all the information required for the process definition activity.

Future work consists in integrating present approaches with feature-based process planning, based on either the import of feature data directly from CAD models or manual input of feature parameters [13].

Manual input can become quite complex and also has the additional overhead of maintaining the feature library or feature descriptions. There are currently many choices for creating feature models in a geometric modelling context. The most common and traditional approaches include 'automatic feature recognition', 'interactive feature definition/identification', and 'design-by-features' [4]. These approaches are generally application specific and have numerous drawbacks and limitations. Editing these feature-oriented models also presents a challenge, as the entire feature tree has to be revalidated after each parameter change.

Providing the user with a tool to create and maintain the feature library will help to overcome the domain/application specific problems of feature-based design. An attempt was also made to represent this information in a generic and unambiguous manner using the STEP application protocols.

\section{Conclusions}

This paper is a contribution to the subject of process planning. The initial approach of the paper is focused on drawing operations in sheet metal processes. A new computer system to help process planning has been developed based on the latest software engineering technology and a comprehensive literature survey as well as research into the requirements and experience of professional users.

The incorporation of many planners' knowledge into the computer system has made the developed system more competent than those that only use one or a few people's knowledge. The system has been tested with typical data provided by the collaborators and the results obtained and feedback received from engineers in the company has shown that the system is much quicker, more efficient and consistent in producing sheet metal fabrication process plans than existing systems reviewed.

The system is flexible and generic as it can be adapted to the customer's needs without making many changes to the code. The system has an intuitive and user-friendly interface.

It should be noted that although the number of forming processes available in the system is limited, further development of the system will include other types of forming processes. The future addition of a standalone feature modeller will allow a different and more graphical method of entering component 
information input into to the system. It will also allow the user to make quick and easy alterations.

Finally, as it is already mentioned in this article, the study has certain limitations that must be taken into account. First of all, in the field of sheet metal processes there are many kinds of operations and the study has focused on drawing operations. Secondly, the computer system is applied to rotational part with one geometrical drawing. Thirdly, it is impossible to ensure that an application can provide the solution in all practical cases.

It would be of considerable interest to continue these studies, not only in relation to the remaining geometry, but also to other existing kinds of sheet metal operations. However, it is believed that by carefully selecting the pieces used in the design of the system, it has obtained, for drawing operations, excellent results in the vast majority of cases studied. The methodology followed here is perfectly applicable to the majority of the sheet metal processes and, consequently, the effort required for continued study in this area is now greatly reduced.

\section{Acknowledgements}

This work was made during a stay in Cranfield University. This stay was sponsored with the 2002BEA/400133 reference grant, which was given by the Catalonian government.

\section{References}

[1] H. Steudel, Past, present and future the CAPP systems, Int. J. Product. Res. 22 (2) (1984) 253-266.
[2] C. Granville, Computer aided process planning, Comput. Aided Eng. 8 (8) (1995) 46-48.

[3] R. Jagirdar, V.K. Jain, J.L. Batra, Characterization and identification of forming features 3D for sheet metal components, Int. J. Mach. Tools Manuf. 41 (9) (2001) 1295-1322.

[4] J.X. Gao, Y.S. Tang, K. Sharma, A feature model editor and process planning system for sheet metal products, J. Mater. Process. Technol. 107 (1) (2000) 88-95.

[5] W.M. Sing, K.P. Rao, Knowledge based process lay-out system for axisymmetrical deep drawing using decision tables, Comput. Ind. Eng. 32 (2) (1997) 299-319.

[6] L. Alting, H. Zhang, Computer aided process planning: the state-of-the art survey, Int. J. Product. Res. 27 (4) (1989) 553-585.

[7] V.V. Kryssanov, A.S. Kleshchev, Y. Fukuda, K. Konishi, Building a logical model in the machining domain for CAPP expert systems, Int. J. Product. Res. 36 (4) (1998) 939-956.

[8] J. Ciurana, G. Romeu, M.L. Castro, R. De, M. Albertí, A system based on machined volumes to reduce the number of route sheets in process planning, Comput. Ind. 51 (1) (2003) 41-50.

[9] R. Radhakrishnan, A. Amsalu, M. Kamran, B.O. Nnaji, Design rule checker for sheet metal components using medial axis transformation and geometric reasoning, J. Manufact. Syst. 15 (3) (1996) 179-189.

[10] J.C. Choi, B.M. Kim, H.Y. Cho, C. Kim, J.H. Kim, An integrated CAD system for the blanking of irregular-shape sheet metal products, J. Mater. Process. Technol. 83 (2) (1998) 84-97.

[11] R. Singh, G.S. Sekhon, An intelligent system for optimal selection of dies and tool for sheet metal operations, J. Eng. Manufact. 217 (2002) 821-828.

[12] C. Wick, R.F. Veilleux, Tool and Manufacturing Engineers' Handbook, 2, Forming. SME, New York, 1990.

[13] C.H. Wang, D.A. Bourne, Design and manufacturing of sheet metal parts: using features to aid process planning and resolve manufacturability problems, Robot. Comput. Integrat. Manufact. 13 (3) (1997) 281-294. 